\title{
Inhibition of Tomato Early Blight Disease by Culture Extracts of a Streptomyces puniceus Isolate from Mangrove Soil
}

\author{
Lingyun Hao, ${ }^{1,2}$ Xiaoli Zheng,,$^{1,2}$ Yu Wang, ${ }^{2}$ Shuangfei Li, ${ }^{2}$ Chenjing Shang, ${ }^{2}$ and Ying $\mathrm{Xu}^{1,2, \dagger}$ \\ ${ }^{1}$ Guangdong Provincial Key Laboratory for Plant Epigenetics, College of Life Sciences and Oceanography, Shenzhen University, Shenzhen \\ 518060, People's Republic of China \\ ${ }^{2}$ Shenzhen Key Laboratory of Marine Bioresource \& Eco-Environmental Science, Shenzhen Engineering Laboratory for Marine Algal \\ Biotechnology, College of Life Sciences and Oceanography, Shenzhen University, Shenzhen 518060, People's Republic of China \\ Accepted for publication 19 February 2019.
}

\begin{abstract}
This study investigates the potential of natural products derived from a mangrove rhizosphere bacterium in tomato early blight management. A Streptomyces puniceus strain L75 was isolated from the rhizosphere of Acanthus ilicifolius Linn in the Mai Po Reserve, Hong Kong. The crude ethyl acetate (EA) extract of L75 fermentation cultures has broadspectrum antifungal bioactivities. L75 EA extract was significantly more effective in Alternaria solani growth inhibition at $25 \mu \mathrm{g} / \mathrm{ml}$ or lower compared with Mancozeb, with no observable negative impacts on tomato leaves or root development. Furthermore, L75 EA extract had

A. solani conidia, with a fungistatic mode of action. Liquid chromatographyquadrupole time-of-flight mass spectrometry analysis identified two possible antifungal compounds, Alteramide A and the Heat-Stable Antifungal Factor, which together contribute partially to the bioactivity of L75 EA extract. On detached tomato leaves, coinoculation of A. solani with L75 EA extract of 50, 25 , or $5 \mu \mathrm{g} / \mathrm{ml}$ reduced diseased areas by $\sim 98, \sim 90$, and $\sim 48 \%$, respectively, relative to the control after 5 days. This study demonstrates the potential of natural products from mangrove rhizosphere bacteria in agricultural applications.
\end{abstract} significantly lower aquatic toxicity than Mancozeb at the same concentrations. L75 EA extract targets germ tube elongation of

Alternaria solani is a wide host-ranged necrotrophic fungal pathogen that infects many important economic crops of the Solanaceous families. On tomato (Solanum lycopersium) plants, the fungus causes early blight, one of the most devastating diseases threatening tomato fruit production in tropical and subtropical areas (Adhikari et al. 2017). Under warm and humid conditions, which are favorable for disease development, the pathogen can infect all parts of the tomato plants, including leaves, stems, and fruits, during the entire plant developmental stage, resulting in symptoms such as defoliation, drying off of twigs, premature fruit drop, and fruit rot (Adhikari et al. 2017). In certain regions, such as Nigeria, the United States, and India, annual economic loss owing to tomato early blight has been estimated up to $>70 \%$ (Chaerani and Voorrips 2006). Because of high genetic variations in pathogenic isolates, a relatively short time needed for sporulation, a prolonged active disease cycle, and a broad host range, A. solani is very difficult to manage (Adhikari et al. 2017; Meena et al. 2017).

Currently, control strategies for managing tomato early blight include cultural practices, fungicide application, and breeding for resistant varieties (Adhikari et al. 2017; Foolad et al. 2008). Because of issues, such as the lack of major resistance genes for early blight in tomato cultivars with market desirable traits and the complex

${ }^{\dagger}$ Corresponding author: Y. Xu; boxuying@szu.edu.cn

Funding: This work was financially supported by Shenzhen Science and Technology Innovation Program [2019] No. 33, National Natural Science Foundation of China grants 31801788 and 41706137, Guangdong Innovation Research Team Fund grant 2014ZT05S078, Shenzhen Science and Technology Innovation Basic Research Project Fund grant JCYJ20170302144706218, and Scientific and Technical Innovation Council of Shenzhen grants KQJSCX20170330110206042 and KQJSCX20170727101743831.

*The $\boldsymbol{e}$-Xtra logo stands for "electronic extra" and indicates that three supplementary figures are published online.

The author(s) declare no conflict of interest.

(C) 2019 The American Phytopathological Society
Keywords: disease control and pest management nature of inheritable quantitative resistance in tomato cultivars, fungicide application remains the major approach to combat this disease. Protective fungicides, such as Mancozeb, and systemic fungicides, such as azoxystrobin with multisite mode of action, are widely used around the world. Several rounds of fungicide application are often required during the tomato growing season to achieve a satisfactory level of disease control (Abu-El Samen et al. 2015). There have been increasing reports on loss of efficacy of commercial fungicides against the pathogen (Abu-El Samen et al. 2016; Gudmestad et al. 2013; Odilbekov et al. 2016). The rise of fungicide-resistant $A$. solani isolates poses a threat to the sustainability of tomato production and warrants studies to search for new antifungal compounds. Bioactive natural products are generally considered environmentally friendly, and thus, they might be considered as sustainable alternatives to synthetic fungicides or may be used in combination with existing fungicides in integrated pest management (Xu et al. 2011).

A number of studies have reported natural products from potential biological control agents, such as certain Bacillus spp. isolates and Chaetomium globosum, as well as the plant extracts poacic acid and Zimmu leaf extracts. These natural products have antifungal bioactivities and are able to inhibit early blight development in planta to different extents (Ali et al. 2016; Latha et al. 2009; Piotrowski et al. 2015; Zhao et al. 2017). These implicate the potential of natural products for disease management in the future, particularly for the organic tomato production system. As a distinct and yet underexplored habitat, the mangrove wetland is renowned for supporting highly diversified microbial organisms with unique species and metabolic adaptations, and thus, it has continuously been a promising resource for novel bioactive compound discovery in the past decade (Ancheeva et al. 2018; de Souza Sebastianes et al. 2013). In a previous study, we established a library of bacterial isolates collected from the rhizosphere soil associated with Acanthus ilicifolius Linn, a common mangrove plant present with great abundance in the Mai Po Reserve, Hong Kong (L. Hao, Y. Wang, X. Chen, X. Zheng, S. Chen, S. Li, Y. Zhang, and Y. Xu, 
unpublished data). We then screened for candidates that produce antifungal compounds with the potential to be explored as environmentally compatible biopesticides. An isolate L75 was found to inhibit the growth of several fungal pathogens in vitro. Initial liquid chromatography-mass spectrometry analysis suggested a rich profile of compounds produced in L75 fermentation cultures.

In this study, we sought to further investigate the potential of the compounds produced by L75 as biopesticides in plant disease management and identify the major bioactive components. We chose to study the $A$. solani-tomato system, because early blight is one of the major threats to tomato production in many regions of China (Song et al. 2015) and because current disease management relies heavily on fungicide application, with no major resistance gene identified (Adhikari et al. 2017). We examined pathogen inhibition efficacies, toxicities, and disease suppression effects of the crude ethyl acetate (EA) extract of L75 fermentation cultures compared with the commercial fungicide Mancozeb. We also investigated the mode of action of L75 EA extract on A. solani conidial germination. Finally, we identified two possible known antifungal compounds that contribute partially to its fungal inhibition bioactivity. This study provides useful information on potential application of natural products from mangrove rhizosphere bacteria in tomato early blight management.

\section{MATERIALS AND METHODS}

Bacterial isolation and identification. A bacterial library consisting of 113 strains isolated from the rhizosphere soil associated with naturally occurring A. ilicifolius $\mathrm{L}$. in a mangrove located in the Mai Po Reserve, Hong Kong was established in a separate study using the methods as described by Ma et al. (2016). An isolate, L75, was found to produce antifungal compounds during the bioactivity screening tests done as described by Lin et al. (2017), and thus, it was chosen for additional investigation (unpublished data). Identification of L75 was conducted through 16s ribosomal DNA (rDNA) sequencing using the universal primers 27F and 1492R (Lin et al. 2017) and blasted with the sequences of known species in the National Center for Biotechnology Information database (https:// www.ncbi.nlm.nih.gov/BLAST/). Isolate L75 16s sequences mostly matched Streptomyces puniceus (100\% similarity; accession number KP209438.1). L75 was routinely cultured at $28^{\circ} \mathrm{C}$ on Marine Agar 2216 (Becton, Dickinson and Company) medium, which is a rich medium commonly used for fast-growing heterotrophs.

Small-scale fermentation and natural products extraction. S. puniceus isolate L75 was grown in medium of $5 \mathrm{~g}$ of glucose, $5 \mathrm{~g}$ of soluble starch, $1 \mathrm{~g}$ of tryptone, $1 \mathrm{~g}$ of peptone, $1 \mathrm{~g}$ of yeast extract, and $17 \mathrm{~g}$ of sea salts per liter of deionized water (SGTPY medium) at $25^{\circ} \mathrm{C}$ and shaken at $200 \mathrm{rpm}$. After 4 days, an equal volume of EA was added into the fermentation cultures and mixed well (Ross et al. 2013). Then, the aqueous layer was discarded, and the EA was removed with a LABOROTA 4000-efficient rotary evaporator under reduced pressure (120-20 mbar; Heidolph Instruments) and a CentriVap (Labconco Corp.). A single crude extract of the metabolites of L75 was obtained and prepared as a $25 \mathrm{mg} / \mathrm{ml}$ stock solution in dimethyl sulfoxide (DMSO) (Lin et al. 2017). This single crude extract was used for assays, including in vitro fungal growth inhibition, toxicities, mode of action, and disease suppression in detached tomato leaves.

Fungal culture and plant growth conditions. Plant fungal pathogens A. solani HLJ65, Setosphaeria turcica St28A, and Cochliobolus heterostrophus Ch16 provided by the laboratory of Zonghua Wang at Minjiang College (Fuzhou, China) as well as Magnaporthe oryzae P131 provided by the laboratory of Youliang Peng at China Agricultural University (Beijing, China) were routinely cultured on potato dextrose agar (PDA) plates (Dickinson and Company) at $28^{\circ} \mathrm{C}$. Tomato cultivar Micro-Tom (MySeeds Company) plants were used in all plant assays, and they were grown using a peat-based potting mix without mycorrhizae (Shengsheng Agricultural Co. Ltd.) at $25^{\circ} \mathrm{C}$ with a 16-h photoperiod as previously described (Song et al. 2015). Half-strength Hoagland's nutrient solution (Song et al. 2015) was applied when necessary.

In vitro fungal growth inhibition assay. To quantify the inhibitory effects of crude L75 EA extract on fungal growth, 4-mmdiameter mycelial plugs of HLJ65, St28A, and Ch16 were taken from 4-day-old PDA cultures using a glass tube and placed individually upside down onto PDA plates supplemented with 50,25, 5, or $1 \mu \mathrm{g} / \mathrm{ml}$ L75 EA extract. For plates supplemented with Mancozeb (Kelilong Biochemical Co. Ltd.), the following concentrations were included: $2 \mathrm{mg} / \mathrm{ml}$ and $50,25,5$, and $1 \mu \mathrm{g} / \mathrm{ml}$. Plates were incubated at $28^{\circ} \mathrm{C}$ for $96 \mathrm{~h}$ for HLJ65 and Ch16 and $120 \mathrm{~h}$ for St28A and P131.

The diameter of each colony was measured twice in two directions that were vertical to each other, and the average diameter was calculated. PDA plates supplemented with DMSO of the same volume $(0.1 \%)$ as used in the L75 EA extract treatments were included as negative controls. Inhibition rate of each treatment was calculated as percentage of inhibition $=100 \% \times($ diameter of control diameter of treatment)/(diameter of control). Three biological replicates were included for each treatment within each experiment, and the experiment was repeated three times for each pathogen.

Phytotoxicity assay. Tomato plants of $\sim 3$ weeks old with approximately three to four true leaves were sprayed weekly with $5 \mathrm{ml} \mathrm{DMSO}(0.1 \%)$ or 25,5 , or $1 \mu \mathrm{g} / \mathrm{ml} \mathrm{L75} \mathrm{EA} \mathrm{extract} \mathrm{for} 4$ weeks and kept at $25^{\circ} \mathrm{C}$ for continuous observation. Pictures were taken 0 , $7,14,21$, and 28 days after application to record leaf and shoot developmental status. Five plants were included in each treatment, and the experiment was repeated three times.

Impacts of L75 EA extract on tomato root development. The effects of L75 EA extract on tomato seedling development were evaluated as previously described with modifications (Stanga et al. 2013). Briefly, within 30 days of harvest, seeds were surface sterilized with $15 \%$ Clorox for $15 \mathrm{~s}$ and then, rinsed thoroughly with sterile water at least four times. Seeds were placed in sterile water for $48 \mathrm{~h}$ at room temperature with shaking to facilitate germination, and then, they were transferred to one-half strength Murashige and Skoog medium (1/2 MS; Caisson Laboratories Inc.) plates and incubated at $25^{\circ} \mathrm{C}$ for 3 days with a $16-\mathrm{h}$ photoperiod. Seedlings with similar root length were then transferred to $1 / 2 \mathrm{MS}$ plates supplemented with DMSO $(0.1 \%)$ or 25 or $5 \mu \mathrm{g} / \mathrm{ml} \mathrm{L75} \mathrm{EA} \mathrm{extract,}$ and they were marked for the initial root length; plates were kept vertical at $25^{\circ} \mathrm{C}$ for another 7 days. Root growth during the second stage was measured using ImageJ. Nine seedlings were included for each treatment, and the experiment was repeated three times.

Aquatic toxicity assay. Algae are a major microbial group that forms the basis of many food webs in the aquatic ecosystems. Furthermore, they are considered as the indicators of acute toxicity of pesticides to nontarget organisms in aquatic systems (Staley et al. 2015). We thus determined the toxicity of L75 EA extract to algae as the first step to examine its environmental impacts. An algae, Chlorella salina, was acclimated in the $\mathrm{f} / 2$ medium $(\mathrm{pH}=8.0)$ in $125-\mathrm{ml}$ flasks at $22^{\circ} \mathrm{C}$ with a $14: 10$-h cycle (light:dark) as previously described (Dong et al. 2013). The initial population of $C$. salina was adjusted to $5 \times 10^{4}$ cells per milliliter with a hemocytometer; supplemented with DMSO $(0.1 \%)$ or 25,5 , or $1 \mu \mathrm{g} / \mathrm{ml}$ L75 EA extract and Mancozeb; and cultured in 24-well plates with a total volume of $1 \mathrm{ml}$ of cell mixture per well. Therefore, each well started with the same population of $C$. salina ( 50 cells per microliter) at time 0 . A $100-\mu \mathrm{l}$ aliquot was sampled every $24 \mathrm{~h}$ for 7 days for population determination by flow cytometry (Beckman Coulter Co. Ltd.) following the manufacturer's instructions and standard operations. Three replicates were included for each treatment, and the experiment was repeated twice.

Mode of action of L75 EA extract. The effects of L75 EA extract on A. solani HLJ65 conidial germination and germ tube development were determined as previously described (Pasche et al. 2005). Briefly, fungal colonies were grown for $\sim 7$ days on PDA 
plates, and conidial production was induced by wounding the mycelium once as previously described (Peng and Shishiyama 1988). Ten days after the wounding, conidia were harvested and adjusted to a concentration of $\sim 1.6 \times 10^{5}$ conidia per milliliter with sterile $\mathrm{H}_{2} \mathrm{O}$. Aliquots of this conidial suspension were supplemented with DMSO $(0.1 \%)$ or 25 or $5 \mu \mathrm{g} / \mathrm{ml} \mathrm{L75} \mathrm{EA} \mathrm{extract.} \mathrm{Then,}$ $10-\mu 1$ droplets of the control (DMSO) and treatment group (with L75 extract) were individually spread onto a microscopic glass slide, which was then placed on top of moist paper towels sealed in a petri dish and incubated at $28^{\circ} \mathrm{C}$ for $6 \mathrm{~h}$. The percentage of germination rate was calculated for every 100 spores counted under a light microscope. Three replicate slides were included for each treatment per experiment, and the experiment was repeated three times. To determine germ tube length, a $200-\mu$ l aliquot of conidial suspension $\left(\sim 5 \times 10^{5}\right.$ spores per milliliter) was plated onto water agar $(1.5 \%)$ plates supplemented with DMSO $(0.1 \%)$ or 25 or $5 \mu \mathrm{g} / \mathrm{ml}$ L75 EA extract and incubated at $28^{\circ} \mathrm{C}$ for $18 \mathrm{~h}$. Pictures were taken with a dissecting microscope, and the germ tube lengths of at least 150 spores were measured with ImageJ.

Determination of whether L75 EA extract has a fungistatic or fungicidal mode of action was done as previously described (Ali et al. 2016). Briefly, $1 \mathrm{ml}$ of $A$. solani HLJ65 conidial suspension in sterile $\mathrm{H}_{2} \mathrm{O}$ of $\sim 1.0 \times 10^{4}$ spores per milliliter was treated with DMSO $(0.1 \%)$ or $25 \mu \mathrm{g} / \mathrm{ml}$ L 75 EA extract and incubated at $28^{\circ} \mathrm{C}$ for $24 \mathrm{~h}$. Then, one-half of the samples of each treatment were centrifuged, washed four times with potato dextrose broth (PDB), resuspended with $1 \mathrm{ml}$ of fresh PDB (Dickinson and Company), and incubated at $28^{\circ} \mathrm{C}$ for another $24 \mathrm{~h}$. These were designated the "washed" samples. The other one-half of the samples were not processed, and they were incubated in the same way and designated the "unwashed" samples. A 100- $\mu$ l aliquot was taken from each sample for measurement of the absorbance (optical density at $600 \mathrm{~nm}$, $\mathrm{OD}_{600}$ ) in a 96-well plate before and after the wash treatment. Net mycelial growth was calculated by subtracting the absorbance at $0 \mathrm{~h}$ from that of the 24-h interval. Normalized net growth, which is the percentage of the value for the untreated DMSO control, was determined by dividing net growth in each treatment by net growth in the untreated control. Three aliquots were taken for OD readings from each replicate, and three replicates were included for each treatment. The experiment was repeated four times.

Fermentation and bioassay-guided isolation and purification of the antifungal components in L75 EA extract. Twenty liters of L75 was grown in SGTPY medium using multiple 1-liter Erlenmeyer flasks at $25^{\circ} \mathrm{C}$ for 4 days. At the end of fermentation, an equal volume of EA was added to the bacterial cultures, and the EA layer was collected. This procedure was repeated three times. The EA layers were combined, and the solvent was then removed as described before to obtain the crude extract, which is about $3 \mathrm{~g}$. The crude extract was then subjected to flash $\mathrm{C} 18$ column chromatography, eluting with 30,60 , and $100 \%$ aqueous $\mathrm{MeOH}$ to yield three fractions (fractions 1 to 3 ). The fractions were obtained as $25 \mathrm{mg} / \mathrm{ml}$ stocks as described in the previous section and tested in the antifungal activity assays conducted in 24-well plates, where an HLJ65 mycelial plug (4 $\mathrm{mm}$ in diameter) was inoculated into $1 \mathrm{ml} \mathrm{PDB}$ per well supplemented with either $25 \mu \mathrm{g} / \mathrm{ml}$ of each fraction or DMSO $(0.1 \%$; negative control). The active fractions (fraction 3) with observable inhibition to fungal growth after 4 days of treatment were further separated into 50 subfractions on a Sephadex LH-20 column eluted with methanol. The subfractions were also tested in the bioassays as described above, and the active fractions (subfractions 14 to 18) were then subjected to analysis by liquid chromatography-quadrupole time-of-flight mass spectrometry (LC-Q-TOF-MS).

Efficacy of L75 EA extract in tomato early blight inhibition on detached leaves. The first and second true leaves of $\sim 3$-week-old Micro-Tom plants were used for the detached leaf assay as previously described with some modifications (Ali et al. 2016). Briefly, healthy-looking leaves were harvested and rinsed with sterile water three times and blot dried gently. Each true leaf (consisting of three leaflets) was laid on top of two layers of sterile moist paper towels with the petioles wrapped with moist cotton and placed in a 9-cm-diamter petri dish. A 40- $\mu$ l droplet of the following treatments (DMSO [0.1\%] or 50,25 , or $5 \mu \mathrm{g} / \mathrm{ml} \mathrm{L75}$ crude EA extract) was applied to the center of each leaflet, and then, an A. solani HLJ65 mycelial plug $4 \mathrm{~mm}$ in diameter punched from a 7-day-old culture was inoculated to the same area of the above treatments on each leaflet so that it was in direct contact with the chemicals. DMSO (0.1\%)-treated leaflets without the fungus were included as negative controls. Petri dishes were incubated at $25^{\circ} \mathrm{C}$ with a 16-h photoperiod. Necrotic areas were measured 5 days postinoculation. Six leaflets were included for each experiment, and the experiment was repeated three times.

Statistical analysis. All statistical analyses were performed using the R program (version 2.14.1; R Development Core Team, R Foundation for Statistical Computing; http://www.R-project.org). Tukey's honestly significant difference test (based on one-way analysis of variance) was used to compare the differences in the mean values among treatments for the following assays: in vitro fungal growth inhibition (Table 1), root length of tomato seedlings (Fig. 1B), algal populations at day 7 (Fig. 2), and conidial germination rates and germ tube length (Fig. 3). Students' $t$ test was used to compare differences between the mean values of each compound treatment and control group (DMSO) of the pathogenicity assay (Fig. 4), and the washed and unwashed groups of the fungicidal assay. Significance was set at a $P<0.05$ for all aforementioned tests.

TABLE 1. Mean inhibition rates (MIRs) to fungal colony growth of the ethyl acetate (EA) extract of L75 fermentation cultures and Mancozeb ${ }^{v}$

\begin{tabular}{|c|c|c|c|c|c|}
\hline \multirow[b]{2}{*}{ Treatments and pathogensw } & \multicolumn{5}{|c|}{$\operatorname{MIRs}(\%)^{\mathrm{x}}$} \\
\hline & $2 \mathrm{mg} / \mathrm{ml}^{\mathrm{y}}$ & $50 \mu \mathrm{g} / \mathrm{ml}$ & $25 \mu \mathrm{g} / \mathrm{ml}$ & $5 \mu \mathrm{g} / \mathrm{ml}$ & $1 \mu \mathrm{g} / \mathrm{ml}$ \\
\hline \multicolumn{6}{|l|}{ L75 EA extract } \\
\hline St28A & NA & $61.6 \pm 2.0 \mathrm{a}^{\mathrm{z}}$ & $64.3 \pm 6.5 \mathrm{a}$ & $45.4 \pm 3.4 \mathrm{~b}$ & $28.9 \pm 4.2 \mathrm{~b}$ \\
\hline Ch16 & NA & $60.5 \pm 1.8 \mathrm{a}$ & $65.6 \pm 0.5 \mathrm{a}$ & $63.6 \pm 1.1 \mathrm{a}$ & $55.4 \pm 3.5 \mathrm{~b}$ \\
\hline P131 & NA & $100.0 \pm 0.0 \mathrm{a}$ & $100.0 \pm 0.0 \mathrm{a}$ & $100.0 \pm 0.0 \mathrm{a}$ & $100 \pm 0.0 \mathrm{a}$ \\
\hline HLJ65 & NA & $48.6 \pm 2.3 \mathrm{~B}$ & $49.7 \pm 1.4 \mathrm{~B}$ & $40.1 \pm 2.4 \mathrm{BC}$ & $27.8 \pm 2.8 \mathrm{CD}$ \\
\hline \multicolumn{6}{|l|}{ Mancozeb } \\
\hline HLJ65 & $65.2 \pm 8.4 \mathrm{~A}$ & $33.1 \pm 0.0 \mathrm{~B}$ & $17.7 \pm 2.3 \mathrm{DE}$ & $2.8 \pm 1.0 \mathrm{E}$ & $2.8 \pm 1.5 \mathrm{E}$ \\
\hline
\end{tabular}

$\mathrm{v}$ Whole crude extract of L75 fermentation cultures by EA.

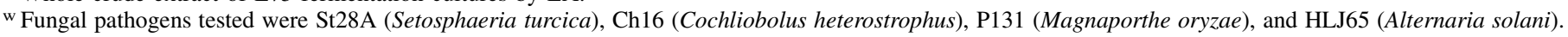

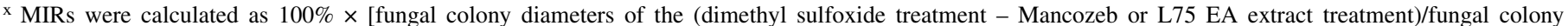
diameters of the dimethyl sulfoxide treatment]; data are shown as the MIRs with standard errors based on 12 replicates from three independent experiments.

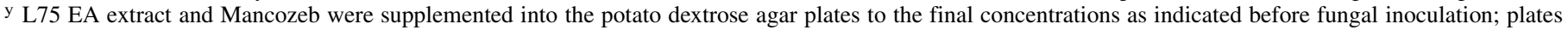
supplemented with dimethyl sulfoxide only of the same volume $(0.1 \%)$ as used in other treatments were included as negative controls. NA: not tested.

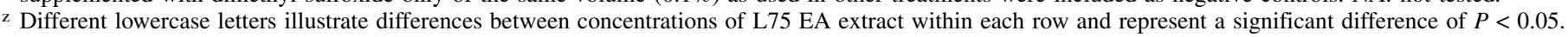
Different uppercase letters illustrate differences between L75 EA extract and Mancozeb at different concentrations in HLJ65 inhibition (comparisons were not made for other pathogens) and represent a significant difference of $P<0.05$. Tukey's honestly significant difference test was used for all comparisons. 


\section{RESULTS}

Bioactivity of L75 EA extracts against plant fungal pathogens. In a previous study, we isolated 113 bacterial strains from the rhizosphere of A. ilicifolius L. from the Mai Po mangrove, Hong Kong and screened for isolates with potential antifungal activities using the crude extracts by EA of their fermentation cultures (data not shown). The EA extract of a strain L75, identified as $S$. puniceus by $16 \mathrm{~s}$ rDNA sequencing (data not shown; accession number KP209438.1), was found to have strong broad antifungal activities against four plant pathogens in vitro at $50 \mu \mathrm{g} / \mathrm{ml}$ (Supplementary Fig. S1). We further quantified the inhibition rates of L75 EA extract at different concentrations. As shown in Table 1, $5 \mu \mathrm{g} / \mathrm{ml} \mathrm{L75}$ EA extract had $\sim 40 \%$ mean inhibition rate (MIR) to A. solani HLJ65 colony growth, which was not significantly different from the 50 - and $25-\mu \mathrm{g} / \mathrm{ml}$ treatments (MIR $=48.6$ and $49.7 \%$, respectively; $P>0.1$ for both). A similar trend was found with L75 EA extract against another pathogen, $C$. heterostrophus Ch16, where $5 \mu \mathrm{g} / \mathrm{ml}$ was equally effective as 25 and $50 \mu \mathrm{g} / \mathrm{ml}$ in fungal growth inhibition, with no significant differences among their MIRs $(P>0.1)$. Regarding $S$. turcica St28A, L75 EA extract of 50 and $25 \mu \mathrm{g} / \mathrm{ml}$ had similar MIRs $(>60 \%)$, whereas $5 \mu \mathrm{g} / \mathrm{ml}$ was less effective $(\mathrm{MIR}=45.4 \% ; P<0.05$ compared with the other two treatments). In addition, L75 EA extract was most effective against the rice blast pathogen P131, with $100 \%$ MIRs even at $1 \mu \mathrm{g} / \mathrm{ml}$. These data suggested that L75 EA extract has broad-spectrum antifungal activities with varying efficacy toward different pathogens.

We also compared the antifungal bioactivities of L75 EA extract against HLJ65, with activity of the commercial fungicide, Mancozeb, which is commonly used in control of tomato early blight worldwide (Abu-El Samen et al. 2015, 2016). Supplemented with a concentration of $2 \mathrm{mg} / \mathrm{ml}$ similar to that used in field trials (Abu-El Samen et al. 2015; Gullino et al. 2010), Mancozeb had $\sim 60 \%$ inhibitory efficacy in plate assays (Table 1 ). However, at the concentration of $50 \mu \mathrm{g} / \mathrm{ml}$, Mancozeb only had $\sim 33 \%$ MIR to HLJ65 growth, which was similar to that of the L75 EA extract at $5 \mu \mathrm{g} / \mathrm{ml}(P>0.1)$. When reduced to $25 \mu \mathrm{g} / \mathrm{ml}$, Mancozeb was significantly less effective in pathogen inhibition than L75 EA extract of the same concentration $(P<0.01)$. Furthermore, Mancozeb was not able to inhibit HLJ65 at $1 \mu \mathrm{g} / \mathrm{ml}(P>0.05$ compared against the expected value of $\mu=0$, indicating no suppression), whereas L75 EA extract still retained $\sim 21.7 \%$ MIR using $1 \mu \mathrm{g} / \mathrm{ml}(P<0.01$ compared with 0$)$. Taken together, these data demonstrated that L75 EA extract is more effective than the commercial fungicide Mancozeb in A. solani HLJ65 growth inhibition at the same concentrations.

Effects of L75 EA extract on plant leaves and roots. Early blight management usually relies on several rounds of foliar fungicide application during the tomato growing season. Thus, it is necessary to investigate whether a potential biopesticide has any negative impacts on plant development. To investigate whether L75 EA extract has phytotoxicity, we sprayed young tomato plants with the crude extract and continuously monitored plant development for 4 weeks. As shown in Figure 1A, weekly foliar application of L75 EA extract up to 28 days at concentrations of 25,5 , and $1 \mu \mathrm{g} / \mathrm{ml}$ did not cause any discoloration of leaves or abnormal shoot growth. No observable differences were seen among plants of different treatments. We also examined the impact of L75 EA extract on root development of tomato seedlings. As shown in Figure 1B, the average root lengths of tomato seedlings grown on $1 / 2 \mathrm{MS}$ plates

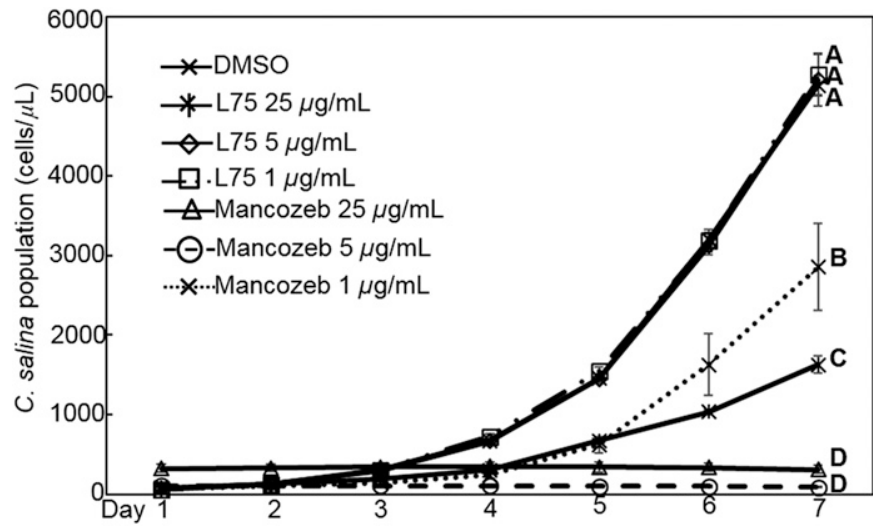

Fig. 2. Toxicity of the ethyl acetate (EA) extract of L75 fermentation cultures and Mancozeb to Chlorella salina. Growth of Chlorella cells treated with 25, 5 , or $1 \mu \mathrm{g} / \mathrm{ml}$ L75 EA extract or Mancozeb. Cells supplemented with dimethyl sulfoxide (DMSO) of the same volume $(0.1 \%)$ as used in the other treatments were included as the control. The averages are based on six replicates from two independent experiments, and the error bars are standard errors. Differences in the mean algal populations at day 7 among treatments were compared using Tukey's honestly significant difference test, and different letters represent a significant difference of $P<0.05$.

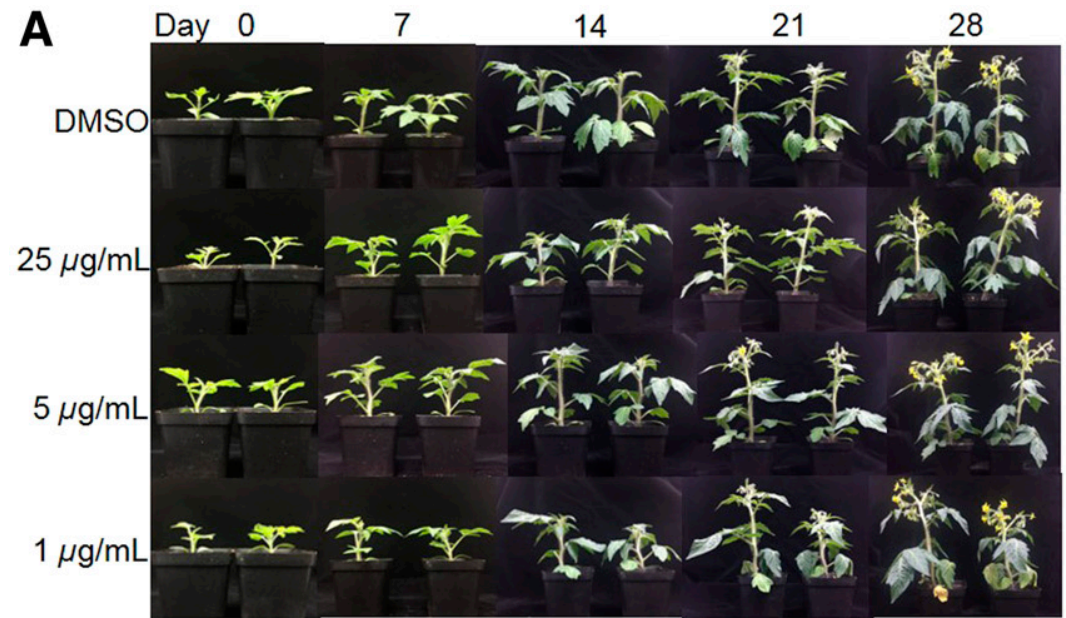

B

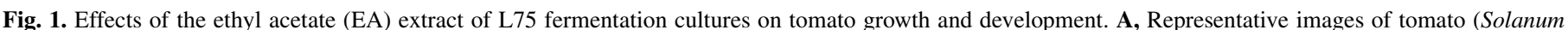

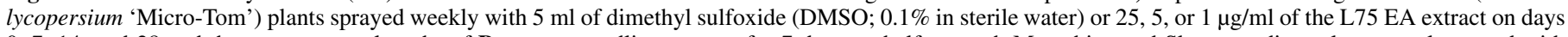

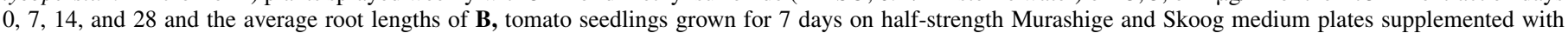

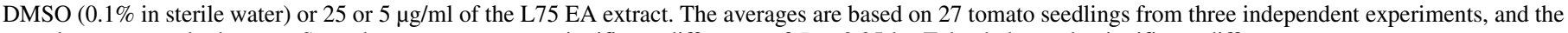
error bars are standard errors. Same letters represent no significant difference of $P>0.05$ by Tukey's honestly significant difference test. 
supplemented with 25 or $5 \mu \mathrm{g} / \mathrm{ml}$ L75 EA extract were $\sim 26$ and $31 \mathrm{~mm}$, respectively, after 7 days, similar to that of the control (average $=29 \mathrm{~mm} ; P>0.1$ ). These results showed that L75 EA extract had no apparent phytotoxicity at the concentrations tested and did not affect root development of young seedlings.

Aquatic toxicity of L75 EA extract. Environmental compatibility of a pesticide is a critical consideration for its real-world application. Pesticide runoff from agricultural land may affect and alter the composition of phytoplankton communities, which in turn, might have a negative impact on the functioning and structure of the whole ecosystem. As a primary producer of the aquatic ecosystems, algae play critical ecological roles, which makes microalgae toxicity tests indispensable for the environmental risk assessment of pesticides (Nyholm and Källqvist 1989). As the initial step to evaluate the environmental toxicities of L75 EA extract, we examined its impact on growth of a beneficial microalgae commonly used in laboratory tests, $C$. salina (Chen et al. 2018). No significant differences were found among the average populations of $C$. salina of the control group (DMSO) and those with 25,5 , or $1 \mu \mathrm{g} / \mathrm{ml} \mathrm{L75} \mathrm{EA} \mathrm{extract} \mathrm{during} \mathrm{the} \mathrm{first}$ $72 \mathrm{~h}$ (Fig. 2). At 7 days posttreatment, the average population of C. salina in the control group was $\sim 5,100$ cells per microliter, significantly higher than that treated with $25 \mu \mathrm{g} / \mathrm{ml}$ L75 EA extract $(\sim 1,600$ cells per microliter; $P<0.01)$. Meanwhile, $C$. salina treated with 5 or $1 \mu \mathrm{g} / \mathrm{ml} \mathrm{L75}$ EA extract had average population sizes of $\sim 5,210$ and $\sim 5,274$ cells per microliter, respectively, with no significant difference from that of the control $(P>0.1$ for both). In contrast, Mancozeb of 25 and $5 \mu \mathrm{g} / \mathrm{ml}$ completely suppressed algal growth, with no increase in population size over the duration of the experiment. Therefore, L75 EA extract has much lower aquatic toxicity than Mancozeb at the same concentrations.

Mode of action of L75 EA extract. To investigate the inhibitory mechanism of L75 EA extract on fungal growth, we examined the impact on A. solani conidial germination and germ tube elongation. After $6 \mathrm{~h}$ of treatment, HLJ65 conidia treated with 25 or $5 \mu \mathrm{g} / \mathrm{ml}$ L 75 EA extract had average germination rates of $\sim 57$ and $\sim 64 \%$, respectively (Fig. 3), with no significant difference from that of the control group (average $=67 \% ; P>0.1$ for both). However, after $18 \mathrm{~h}$ of treatment, the average germ tube lengths of spores treated with 25 or $5 \mu \mathrm{g} / \mathrm{ml} \mathrm{L75} \mathrm{EA} \mathrm{extract} \mathrm{were} 613$ and $393 \mu \mathrm{m}$, respectively, significantly shorter than that of the control (average $=2,206 \mu \mathrm{m} ; P<0.001$ for both). These data suggested that L75 EA extract inhibited HLJ65 conidial germ tube elongation instead of affecting conidial germination.

Compounds with antifungal activities usually have two modes of action, fungicidal and fungistatic, differentiated by whether the

fungus is able to resume growth after removal of the compound (Ali et al. 2016). As shown in Supplementary Fig. S2, after replacement of the extracts with fresh PDB medium, HLJ65 conidia pretreated with $25 \mu \mathrm{g} / \mathrm{ml} \mathrm{L75} \mathrm{EA} \mathrm{extract} \mathrm{for} 24 \mathrm{~h}$ were able to resume growth $(P=0.01$ between washed and unwashed treatments), although to a reduced level compared with the control $(P<0.01$ between washed DMSO and washed $25 \mu \mathrm{g} / \mathrm{ml}$ L75 EA extract treatments), demonstrating a fungistatic mode of action.

Identification of the antifungal components in L75 EA extract. Bioassay-guided fractionation suggested that subfractions 14 to 18 yielded from fraction 3 of L75 EA extract contained the active antifungal ingredients (data not shown). Three main components were clearly present in these subfractions (Supplementary Fig. S3). Unfortunately, we failed to accumulate enough of the individual compounds for nuclear magnetic resonance spectroscopy analysis to confirm their chemical structures. However, the LC-QTOF-MS analysis did provide us with some important clues about the identity of these compounds. By comparing their ultraviolet/visible absorption spectra and high-resolution molecular weight data with those reported in literature, we found that peaks 1 and 3 were presumably Alteramide A and the Heat Stable Antifungal Factor (HSAF), respectively (Fig. 4).

Inhibition of tomato early blight by L75 EA extract. Because of the low yield of the active components, we tested the efficacy of the whole crude extract on disease inhibition in detached tomato leaves, which is an established ex vivo disease severity test system utilized by others for examination of the bioactivity of biocontrol agents in plants (Ali et al. 2016; Chen et al. 2003; Massawe et al. 2018). Because obtaining bacterial crude extracts through fermentation is relatively cheap but purification of pure

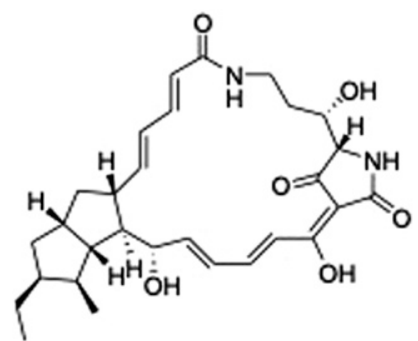

Alteramide A

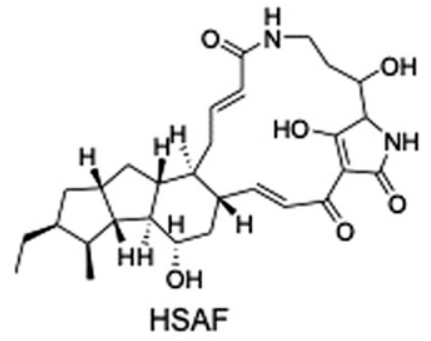

Fig. 4. Chemical structures of the two possible antifungal bioactive components identified from the ethyl acetate extract of L75 fermentation cultures: Alteramide A and the Heat Stable Antifungal Factor (HSAF).
A

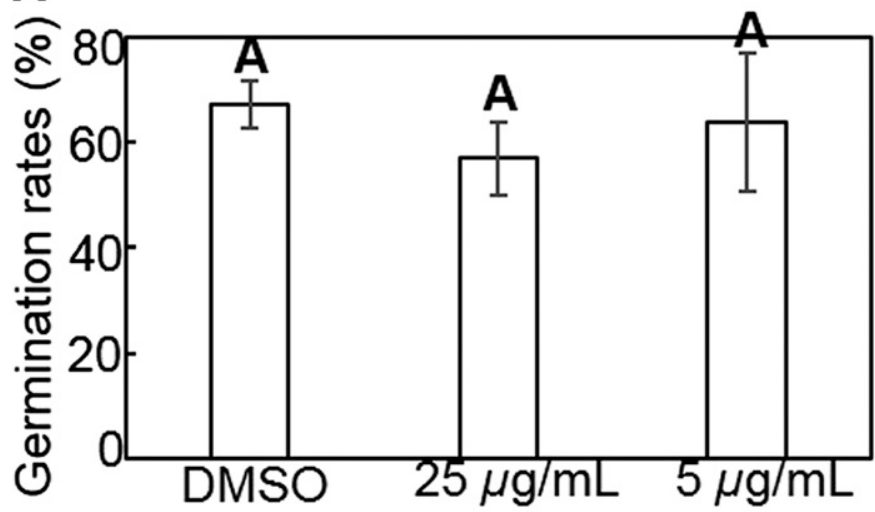

B

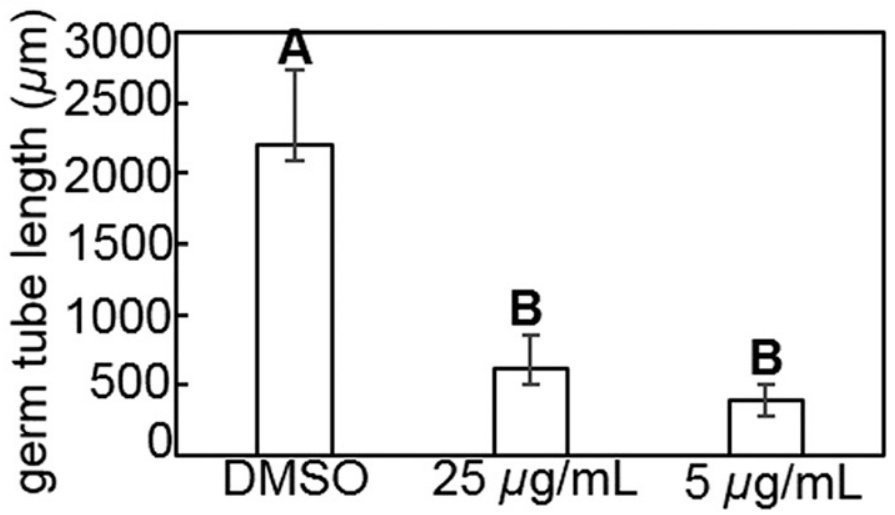

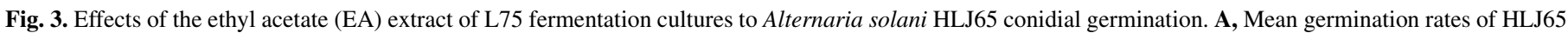

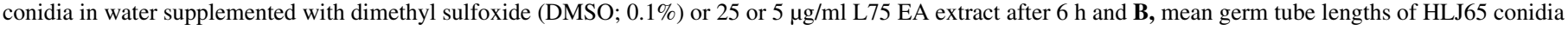

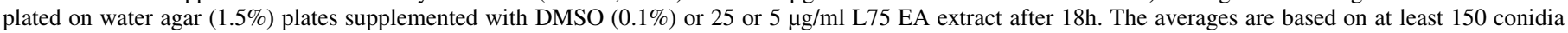

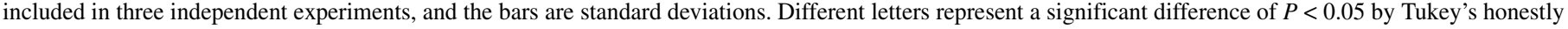
significant difference test. 
compounds could be very costly, it is more economically affordable to use the crude extract instead of pure compounds given that the crude extract poses low or no harm to nontarget organisms. At 5 days postinoculation, control leaves inoculated with the pathogen plus DMSO only showed typical early blight symptoms; greyish necrotic regions developed surrounding the inoculation sites accompanied by discoloration, presumably owing to toxin produced by A. solani, and the average diseased areas were $\sim 58.5 \mathrm{~mm}^{2}$ (Fig. 5). In contrast, necrosis development in the L75 EA extract treatments was significantly suppressed. When 50,25 , or $5 \mu \mathrm{g} / \mathrm{ml}$ L75 EA extract was applied, the average diseased areas of each group were $\sim 1.3, \sim 5.6$, and $\sim 30.2 \mathrm{~mm}^{2}$, respectively, which were all significantly lower than that of the control $(P<0.01$ for all), resulting in $\sim 98, \sim 90$, and $\sim 48 \%$ disease reduction, respectively. Therefore, L75 EA extract was able to suppress early blight development when applied in planta and has the potential to be developed as an environmentally compatible biopesticide.

\section{DISCUSSION}

Mangrove is one of the most unique environments in nature, sharing ecological features with land and ocean, and yet, it is different from both of them. Mangrove soil hosts a large community of microorganisms, which have been a hot area for novel species and natural lead compounds discovery (Azman et al. 2015; Law et al. 2017; Liang et al. 2007; Xu et al. 2014). In the past decade, research on novel bioactive compound identification and characterization from mangrove-associated microbes is on the rise, primarily with medical interests (Ancheeva et al. 2018; Thatoi et al. 2013). However, research on exploring natural products from such resources in agricultural application remains understudied. In a previous study, our laboratory screened a bacterial library containing $>100$ strains isolated and identified from the rhizosphere soil associated with a native mangrove plant A. ilicifolius L. in Maipo Reserve, Hong Kong and discovered that $>25 \%$ of the isolates could produce antimicrobial compounds varying in target

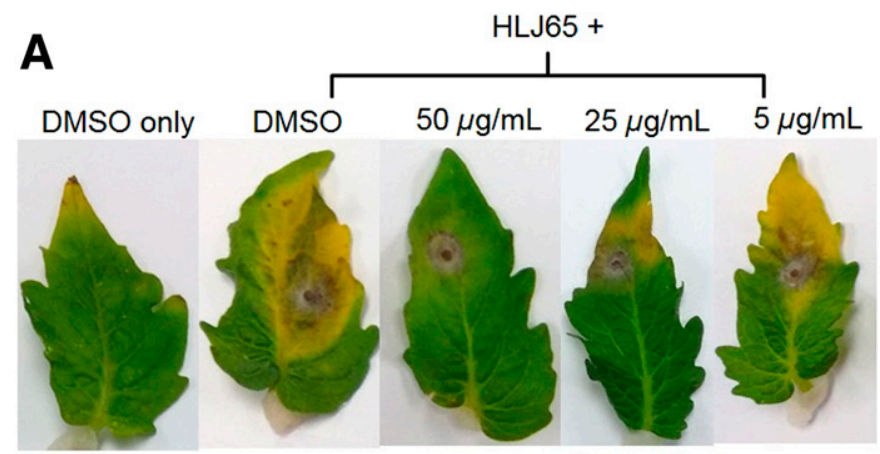

B

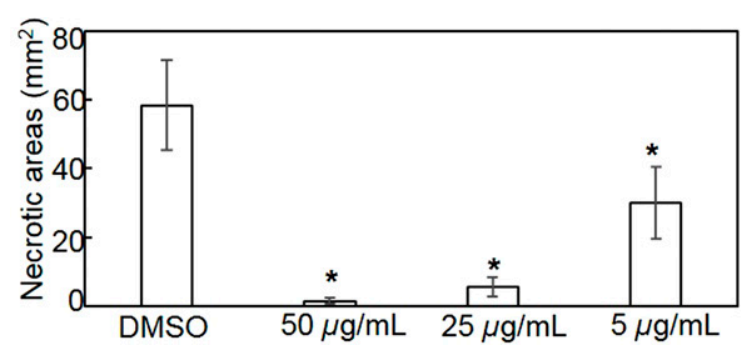

Fig. 5. Inhibition of early blight by the ethyl acetate (EA) extract of L75 fermentation cultures on detached tomato leaves. A, Representative images and $\mathbf{B}$, quantification of necrotic areas induced by Alternaria solani (HLJ65) in detached tomato leaflets treated with $40 \mu$ of dimethyl sulfoxide (DMSO; $0.1 \%$ ) or 50,25 or $5 \mu \mathrm{g} / \mathrm{ml} \mathrm{L} 75$ EA extract at 5 days postinoculation. Leaflets spotted with DMSO only were included as the negative controls. The averages are based on 18 leaflets from three independent experiments, and the error bars are standard errors. * indicates significant difference of $P<0.05$ by Student's $t$ test. specificity and inhibitory efficacy against a variety of important plant pathogens, including $C$. heterostrophus, M. oryzae, A. solani, Colletotricum fructicola, Pseudomonas syringae pv. tomato DC3000, and Pectobacterium carotovorum (unpublished data), demonstrating the potential of mining bioactive natural products from this environment for plant disease management.

The strain L75 has been identified as $S$. puniceus by 16 s rDNA sequencing. Previously, an S. puniceus strain AS13, isolated from the northwestern Himalayas, was reported to produce a bioactive compound Dinactin, which has antibacterial activities against a number of gram-positive and gram-negative bacteria as well as antitumor activities but with low cell toxicity (Hussain et al. 2018). To the best of our knowledge, no $S$. puniceus strains have previously been reported to produce antifungal metabolites. The crude L75 EA extract inhibits colony growth of several important plant pathogens of the Ascomycota. Additional examination of L75 EA extract on fungal development revealed that the crude extract inhibits $A$. solani conidia germ tube elongation but has no effect on conidial germination, with a fungistatic mode of action. These data suggested that the L75 EA extract may be used as a protective fungicide, and several applications might be needed during the tomato growth seasons to achieve optimal management results for early blight. On detached tomato leaves, $25 \mu \mathrm{g} / \mathrm{ml}$ L75 EA extract resulted in $\sim 90 \%$ disease reduction, which is comparable with that of the commercial fungicide, such as Mancozeb at $2 \mathrm{mg} / \mathrm{ml}$, in the field toward a variety of Alternaria isolates (Abu-El Samen et al. 2015). This is consistent with the in vitro bioactivity test results that L75 EA extract was much more effective than Mancozeb in pathogen growth inhibition at the same concentrations. We conducted the pathogenicity assay with mycelial plugs, which have been utilized by other studies to determine the efficacies of the biocontrol agents in disease suppression (Ali et al. 2016; Massawe et al. 2018). We are aware that inoculum type, such as mycelial plugs versus conidia, might affect the efficacy of L75 EA extract in planta. Because L75 EA extract did not cause any observable phytotoxicity in tomato plants, additional investigation of the disease suppression efficacy of the extract by foliar application in whole tomato plants inoculated with Alternaria conidia and testing of the impacts of application frequency and timing on disease control results would be necessary. Furthermore, germinating conidia can rapidly penetrate leaf and may avoid fungicides that remain on the leaf surface. Compounds extracted by EA commonly include small molecules of low to medium polarity, which can readily diffuse into plant cells (Riederer and Friedmann 2006; Schreiber 2005). Thus, there is a good chance that L75 EA extract can be absorbed into plants, providing additional protection against fungal infections or exhibiting other modes of actions, such as induced resistance, which need additional verification. Studies on compound stability and efficacy impacted by environmental factors, such as temperature and ultraviolet radiation, would also be helpful for its future development as a potential biopesticide.

LC-Q-TOF-MS analysis of the active components of L75 EA extract identified two possible known tetramic acid-containing macrolactams, Alteramide A and the HSAF. The former one is a tetracyclic alkaloid that was first isolated from an Alteromonas sp. associated with the Marine Sponge Halichondria okadai (Shigemori et al. 1992), and the HSAF was isolated from the bacterium Lysobacter enzymogenes strain $\mathrm{C} 3$, which is a biological control agent capable of inhibiting diseases caused by several fungal pathogens (Yu et al. 2006). It is not a surprise to identify identical compounds from distantly related bacteria because of high frequencies of genetic material exchange occurrence in nature, and microorganisms are known to share a significant number of homologous genes. The primary compound of the heat-stable antifungal activity in strain C3 was identified as dihydromaltophilin, an antibiotic first reported in a Streptomyces sp. (Graupner et al. 1997). Dihydromaltophilin has been reported to have $\sim 75$ to $89 \%$ of disease control caused by a grape fungal pathogen Plasmopara viticola when applied at $25 \mathrm{ppm}$ in the protectant test, whereas it is not as effective in the curative test. This result is consistent with our hypothesis that L75 
EA extract can be used as a protective fungicide. HSAF targets the biosynthesis of sphingolipids, which are ubiquitous components of eukaryotic cell membranes, and inhibits germination of Bipolaris sorokiniana spores (Li et al. 2008). This is different from our results: that L75 EA extract has no effects on A. solani germination but inhibits subsequent germ tube elongation. This suggests that the actual concentration of HSAF in L75 EA extract might be lower than that used by Li et al. (2008), because even different bacteria could produce the same compound in different concentrations. It could also be because of the differences in sensitivity of the tested pathogens to the antifungal compounds between the two studies. Moreover, Alteramide A and HSAF together only partially account for the antifungal activity of L75 EA extract. Additional identification of other bioactive components and investigation of the synergistic effects of different components will be useful for the formulation of a commercial product developed from L75 EA extract.

For successful biocontrol function with an antibiosis mechanism, the microbes must establish and persist in the intended niche. A failure to achieve a population level to produce enough compound needed for effective disease suppression would compromise their efficacy. This was seen in the case of the L. enzymogenes strain C3 under field conditions, which failed to suppress brown patch disease possibly because of its lower colonization of fescue canopies compared with some other epiphytic bacteria (Giesler and Yuen 1998). In contrast, bioactive microbial extracts can be applied at known concentrations and frequencies, and thus, relatively good consistence can be achieved in disease management results given that they have good stability under environmental conditions. The cost of obtaining L75 crude extract may not be comparable with Mancozeb at this stage, because we used synthetic medium for laboratory test purposes. However, through optimization of the fermentation process through temperatures, medium composition, and time (Tang et al. 2018), it is possible to further increase the yield of L75 extract and reduce the cost. L75 EA extract contains multiple bioactive components, which might have multiple modes of action, resulting in less likelihood of inducing resistance development in the pathogen. Furthermore, it is much more effective in A. solani inhibition but less toxic to a beneficial alga than the commercial fungicide. These together provide useful information for future development of natural products from L75 as potential environmentally compatible biopesticides in tomato early blight management.

\section{ACKNOWLEDGMENTS}

We thank the former graduate student Ke Ma and the undergraduate student Xiaoting Huang for helping with the phytotoxicity assay.

\section{LITERATURE CITED}

Abu-El Samen, F., Goussous, S. J., Al-Shudifat, A., and Makhadmeh, I. 2016. Reduced sensitivity of tomato early blight pathogen (Alternaria solani) isolates to protectant fungicides, and implication on disease control. Arch. Phytopathol. Plant Prot. 49:120-136.

Abu-El Samen, F. M., Goussous, S. J., Jendi, A. A., and Makhadmeh, I. M. 2015. Evaluation of tomato early blight management using reduced application rates and frequencies of fungicide applications. Int. J. Pest Manage. 61:320-328.

Adhikari, P., Oh, Y., and Panthee, D. R. 2017. Current status of early blight resistance in tomato: An update. Int. J. Mol. Sci. 18:2019.

Ali, G. S., El-Sayed, A. S. A., Patel, J. S., Green, K. B., Ali, M., Brennan, M., and Norman, D. 2016. Ex Vivo application of secreted metabolites produced by soil-inhabiting Bacillus spp. efficiently controls foliar diseases caused by Alternaria spp. Appl. Environ. Microbiol. 82:478-490.

Ancheeva, E., Daletos, G., and Proksch, P. 2018. Lead compounds from mangrove-associated microorganisms. Mar. Drugs 16:319-349.

Azman, A.-S., Othman, I., Velu, S. S., Chan, K.-G., and Lee, L.-H. 2015. Mangrove rare actinobacteria: Taxonomy, natural compound, and discovery of bioactivity. Front. Microbiol. 6:856.

Chaerani, R., and Voorrips, R. E. 2006. Tomato early blight (Alternaria solani): The pathogen, genetics, and breeding for resistance. J. Gen. Plant Pathol. 72:335-347.
Chen, H., Zhang, Z., Tian, F., Zhang, L., Li, Y., Cai, W., and Jia, X. 2018. The effect of $\mathrm{pH}$ on the acute toxicity of phenanthrene in a marine microalgae Chlorella salina. Sci. Rep. 8:17577.

Chen, W. Q., Morgan, D. P., Felts, D., and Michailides, T. 2003. Antagonism of Paenibacillus lentimorbus to Botryosphaeria dothidea and biological control of panicle and shoot blight of pistachio. Plant Dis. 87:359-365.

de Souza Sebastianes, F. L., Romão-Dumaresq, A. S., Lacava, P. T., Harakava, R., Azevedo, J. L., de Melo, I. S., and Pizzirani-Kleiner, A. A. 2013. Species diversity of culturable endophytic fungi from Brazilian mangrove forests. Curr. Genet. 59:153-166.

Dong, F., Li, J., Chankvetadze, B., Cheng, Y., Xu, J., Liu, X., Li, Y., Chen, X., Bertucci, C., and Tedesco, D. 2013. Chiral triazole fungicide difenoconazole: Absolute stereochemistry, stereoselective bioactivity, aquatic toxicity, and environmental behavior in vegetables and soil. Environ. Sci. Technol. 47:3386-3394.

Foolad, M. R., Merk, H. L., and Ashrafi, H. 2008. Genetics, genomics and breeding of late blight and early blight resistance in tomato. Crit. Rev. Plant Sci. 27:75-107.

Giesler, L. J., and Yuen, G. Y. 1998. Evaluation of Stenotrophomonas maltophilia strain C3 for biocontrol of brown patch disease. Crop Prot. 17: 509-513.

Graupner, P. R., Thornburgh, S., Mathieson, J. T., Chapin, E. L., Kemmitt, G. M., Brown, J. M., and Snipes, C. E. 1997. Dihydromaltophilin; a novel fungicidal tetramic acid containing metabolite from Streptomyces sp. J. Antibiotics 50:1014-1019.

Gudmestad, N. C., Arabiat, S., Miller, J. S., and Pasche, J. S. 2013. Prevalence and impact of SDHI fungicide resistance in Alternaria solani. Plant Dis. 97: 952-960.

Gullino, M. L., Tinivella, F., Garibaldi, A., Kemmitt, G., Bacci, L., and Sheppard, B. 2010. Mancozeb: Past, present, and future. Plant Dis. 94: 1076-1087.

Hussain, A., Rather, M. A., Dar, M. S., Dangroo, N. A., Aga, M. A., qayum, A., Shah, A. M., Ahmad, Z., Dar, M. J., and Hassan Q. P. 2018. Streptomyces puniceus strain AS13., production, characterization and evaluation of bioactive metabolites: A new face of dinactin as an antitumor antibiotic. Microbiol. Res. 207:196-202.

Latha, P., Anand, T., Ragupathi, N., Prakasam, V., and Samiyappan, R. 2009. Antimicrobial activity of plant extracts and induction of systemic resistance in tomato plants by mixtures of PGPR strains and Zimmu leaf extract against Alternaria solani. Biol. Control 50:85-93.

Law, J. W.-F., Ser, H.-L., Duangjai, A., Saokaew, S., Bukhari, S. I., Khan, T. M., Ab Mutalib, N.-S., Chan, K.-G., Goh, B.-H., and Lee, L.-H. 2017. Streptomyces colonosanans sp. nov., A novel actinobacterium isolated from Malaysia mangrove Soil exhibiting antioxidative activity and cytotoxic potential against human colon cancer cell lines. Front. Microbiol. 8:877.

Li, S., Jochum, C. C., Yu, F., Zaleta-Rivera, K., Du, L., Harris, S. D., and Yuen, G. Y. 2008. An antibiotic complex from Lysobacter enzymogenes strain C3: Antimicrobial activity and role in plant disease control. Phytopathology 98 : 695-701.

Liang, J.-B., Chen, Y.-Q., Lan, C.-Y., Tam, N. F. Y., Zan, Q.-J., and Huang, L.-N. 2007. Recovery of novel bacterial diversity from mangrove sediment. Mar. Biol. 150:739-747

Lin, H. N., Wang, K. L., Wu, Z. H., Tian, R. M., Liu, G. Z., and Xu, Y. 2017. Biological and chemical diversity of bacteria associated with a marine flatworm. Mar. Drugs 15:281.

Ma, Z. P., Lao, Y. M., Jin, H., Lin, G. H., Cai, Z. H., and Zhou, J. 2016. Diverse profiles of AI-1 type quorum-sensing molecules in culturable bacteria from the mangrove (Kandelia obovata) rhizosphere environment. Front Microbiol. 7:1957.

Massawe, V. C., Hanif, A., Farzand, A., Mburu, D. K., Ochola, S. O., Wu, L., Tahir, HAS., Gu, Q., Wu, H., and Gao, X. 2018. Volatile compounds of endophytic Bacillus spp. have biocontrol activity against Sclerotinia sclerotiorum. Phytopathology 108:1373-1385.

Meena, M., Gupta, S. K., Swapnil, P., Zehra, A., Dubey, M. K., and Upadhyay, R. S. 2017. Alternaria toxins: Potential virulence factors and genes related to pathogenesis. Front. Microbiol. 8:1451.

Nyholm, N., and Källqvist, T. 1989. Methods for growth inhibition toxicity tests with freshwater algae. Environ. Toxicol. Chem. 8:689-703.

Odilbekov, F., Edin, E., Garkava-Gustavsson, L., Hovmalm, H. P., and Liljeroth, E. 2016. Genetic diversity and occurrence of the F129L substitutions among isolates of Alternaria solani in south-eastern Sweden. Hereditas 153:10.

Pasche, J. S., Piche, L. M., and Gudmestad, N. C. 2005. Effect of the F129L mutation in Alternaria solani on fungicides affecting mitochondrial respiration. Plant Dis. 89:269-278.

Peng, Y.-L., and Shishiyama, J. 1988. Temporal sequence of cytological events in rice leaves infected with Pyricularia oryzae. Can. J. Bot. 66:730-735.

Piotrowski, J. S., Okada, H., Lu, F., Li, S. C., Hinchman, L., Ranjan, A., Smith, D., Higbee, A. J., Ulbrich, A., Coon, J. J., Deshpande, R., Bukhman, Y. V., 
Mcllwain, S., Ong, I. M., Myers, C. L., Boone, C., Landick, R., Ralph, J., Kabbage, M., and Ohya, Y. 2015. Plant-derived antifungal agent poacic acid targets $\beta$-1,3-glucan. Proc. Natl. Acad. Sci. USA 112:1490-1497.

Riederer, M., and Friedmann, A. 2006. Pages 250-279 in: Biology of the Plant Cuticle, Vol. 23, M. Riederer and C. A. P. Müller, eds. Blackwell Publishing, Oxford, United Kingdom.

Ross, A. C., Xu, Y., Lu, L., Kersten, R. D., Shao, Z., Al-Suwailem, A. M., Dorrestein, P. C., Qian, P. Y., and Moore, B. S. 2013. Biosynthetic multitasking facilitates thalassospiramide structural diversity in marine bacteria. J. Am. Chem. Soc. 135:1155-1162.

Schreiber, L. 2005. Polar paths of diffusion across plant cuticles: New evidence for an old hypothesis. Ann. Bot. 95:1069-1073.

Shigemori, H., Bae, M. A., Yazawa, K., Sasaki, T., and Kobayashi, J. 1992. Alteramide A, a new tetracyclic alkaloid from a bacterium Alteromonas sp. associated with the marine sponge Halichondria okadai. J. Org. Chem. 57: 4317-4320.

Song, Y., Chen, D., Lu, K., Sun, Z., and Zeng, R. 2015. Enhanced tomato disease resistance primed by arbuscular mycorrhizal fungus. Front. Plant Sci. 6:786.

Staley, Z. R., Harwood, V. J., and Rohr, J. R. 2015. A synthesis of the effects of pesticides on microbial persistence in aquatic ecosystems. Crit. Rev. Toxicol. 45:813-836.
Stanga, J. P., Smith, S. M., Briggs, W. R., and Nelson, D. C. 2013. SUPPRESSOR OF MORE AXILLARY GROWTH2 1 controls seed germination and seedling development in Arabidopsis. Plant Physiol. 163:318-330.

Tang, B., Sun, C., Zhao, Y., Xu, H., Xu, G., and Liu, F. 2018. Efficient production of heat-stable antifungal factor through integrating statistical optimization with a two-stage temperature control strategy in Lysobacter enzymogenes $\mathrm{OH} 11 . \mathrm{BMC}$ Biotechnol. 18:69.

Thatoi, H., Behera, B. C., Mishra, R. R., and Dutta, S. K. 2013. Biodiversity and biotechnological potential of microorganisms from mangrove ecosystems: A review. Ann. Microbiol. 63:1-19.

Xu, D.-B., Ye, W.-W., Han, Y., Deng, Z.-X., and Hong, K. 2014. Natural products from mangrove actinomycetes. Mar. Drugs 12:2590-2613.

Xu, X. M., Jeffries, P., Pautasso, M., and Jeger, M. J. 2011. Combined use of biocontrol agents to manage plant diseases in theory and practice. Phytopathology 101:1024-1031.

Yu, F., Zaletarivera, K., Zhu, X., Huffman, J., Millet, J. C., Harris, S. D., Yuen, G., Li, X. C., and Du, L. 2006. Structure and biosynthesis of Heat-Stable Antifungal Factor (HSAF), a broad-spectrum antimycotic with a novel mode of action. Antimicrob. Agents Chemother. 51:64-72.

Zhao, S. S., Zhang, Y. Y., Yan, W., Cao, L. L., Xiao, Y., and Ye, Y. H. 2017. Chaetomium globosum CDW7, a potential biological control strain and its antifungal metabolites. FEMS Microbiol. Lett. 364:fnw287. 